\title{
Review of twenty-four-hour nursed care
}

\author{
Rob Macpherson \& Bill Jerrom
}

Twenty-four-hour nursed care refers to a model of psychiatric care which in fact varies quite significantly from unit to another. This sort of residential care unit has also been called the "hospital hostel" (Goldberg et al, 1985), "ward in a house" (Wykes \& Wing, 1982), "psychiatric nursing home" and in some cases "staffed group home". Although the unit size, staffing structures and ratios, and the degree of direct management within the National Health Service (NHS) vary, this model of care has some quite specific characteristics, which have been summarised by Shepherd et al (1994) as follows:

"A homely, non-institutional domestic setting, with ideally 6-12 residents being cared for in a geographical location removed from hospital, in a 'normal', usually semi/detached/terraced house. ...Expectations of resident involvement in domestic activities such as cooking, cleaning, housework. ...High quality of professional care provided by intensive staff input, high staffing levels, individualised treatment programmes. ...Good access to community facilities, and sometimes close working with local day care/therapeutic facilities".

Allen et al (1993) reported varied staffing in different units, ranging from 8 to 16 full-time staff providing regular sessions per week per unit (some of this difference explained by differing resident numbers).

\section{Place of 24-hour nursed care for the severely mentally ill}

Care in psychiatric hostels is appropriate for people who have an ongoing need for nursing care and supervision, and generally for patients at higher risk of aggression or serious exploitation, often because of treatment-resistant psychotic illness. This type of care is part of a spectrum of specialised residential care which ranges from, at the least dependent level, group homes/supported flats, in which patients either share houses through a model of communal living or live independently, with varying levels of support. 'Supported lodgings' or 'landlady care schemes' provide care on a private basis, but generally under the auspices of a social services scheme which oversees the staffing and finance within each lodging, and the placement of patients. In many districts there has been a rapid expansion of this type of care, in which lodgings staff are often ex-nurses or people gaining national vocational qualifications in care. Such units vary from small, family-run lodgings to quite large institutions which may care for up to 25 patients. There is a dearth of literature in this area, although the interested reader will find a description of the Gloucestershire scheme in Anstee's (1985) paper. There is inevitably an overlap between care provided in such units and 24-hour nursed care, although Robson (1995) demonstrated the potential value of the Clifton Assessment Procedures for the Elderly Behaviour Rating Scale (CAPE BRS) as an aid to assessing the appropriateness of placements. It is evident that in changing clinical circumstances, movement between different types of care provision is likely to be necessary and beneficial.

\section{National perspective}

The 'new long-stay' patient has been a key concept in understanding the development of the hostel

Rob Macpherson is a Consultant Rehabilitation/ General Psychiatrist and Clinical Tutor at Wotton Lawn Hospital, Horton Road, Gloucester GL1 3WL. His current research interests are in the treatment of schizophrenia and services for patients with severe mental illness. Bill Jerrom is Head of Psychology at United Bristol Healthcare NHS Trust. He also holds Honorary Senior Lecturer posts in the Departments of Experimental Psychology and Psychiatry at the University of Bristol. His main interests are in the area of psychological interventions in severe mental illness, particularly in schizophrenia and psychosis. 
facility. Wing (1971) and Mann \& Cree (1976) developed this term to refer to patients who had stayed in hospital for more than one but less than five years, that is, those patients who despite modern treatment approaches could not be discharged from in-patient settings. Early descriptions of the attempts to move such patients out of hospital in to a "ward in a house" (Wykes \& Wing, 1982) or a "hospital hostel ward" (Goldberg et al, 1985), suggested that patients presenting challenging behaviour with a variety of diagnoses could be effectively managed in such units.

Over the past 15 years 24-hour nursed care outside hospital settings has been developed in association with the psychiatric hospital closure programme. While the majority of patients were rehabilitated from long-stay asylums into largely unsupervised social services group homes or supported lodgings schemes, with varying degrees of paid but unprofessional care, a group of patients with more complex needs, generally associated with challenging behaviour, continues to require specialised psychiatric care and supervision. In his review, Lelliott (1996) demonstrated that in reality few hospital beds have been replaced by other residential facilities in the community, leaving only onethird of the residential places (hostel or hospital) that existed 40 years ago and resulting in great pressure on acute psychiatric services. He argued that Department of Health (1991) guidance to health authorities to commission 5000 beds in facilities staffed by nurses 24 hours a day, was "the right policy".

\section{Effectiveness of 24-hour nursed care}

\section{Pioneering units}

A number of well-designed studies have examined the effectiveness of 24-hour nursed care in the three pioneering UK hostel ward projects; 111 Denmark Hill (The Maudsley, London), Cranbury Terrace (Southampton) and Douglas House (Manchester). The results were summarised recently by Shepherd (1998).

There has been only one small $(n=11)$, randomised controlled trial of placement in hostel versus continuing hospital care (Hyde et al, 1987); over a two-year period hostel patients improved socially and showed less psychiatric deterioration than the control subjects. Costs were mid-way between acute and long-stay hospital care. Throughout the research carried out (to date) in the three units
Box 1. Aims and provision of 24-hour nursed care

Twenty-four-hour nursed care is generally targeted at helping the 'new long-stay' population to move successfully out of hospital into a more 'normal' environment

There is a gross national under-provision of specialised residential facilities for the mentally ill

The Department of Health recommends the provision of a minimum of 10 24-hour nursed beds per 10000 population

mentioned above, the hostel patients have improved more than the control subjects. Most changes occurred in the first six months (Wykes, 1982), particularly in improved social functioning (Gibbons, 1986; Hyde et al, 1987). In a 16-year follow-up Reid \& Garety (1996) found that $42 \%$ of the hostel patients were successfully discharged, an active throughput occurring in all studies. The mean length of stay for the successfully resettled group was 2.5 years at Douglas House (Creighton et al, 1991) and three years at the The Maudsley (Garety et al, 1988). Gibbons \& Butler (1987) found that hostel residents believed they had more freedom, none of their sample wishing to return to hospital care.

\section{Subsequent studies}

Further studies in the UK and other countries, have investigated various aspects of hostel care and its relative value compared with ongoing hospital care, and the results are summarised in Table 1 . The research is varied and heterogeneous, reflecting different clinical approaches in the units described.

Box 2. Clinical outcome of 24-hour nursed care

Most patients selected for transfer from hospital to psychiatric hostels are successfully placed.

A small proportion has to be readmitted, generally owing to problems of aggression/ antisocial behaviour.

All hostels appear to have a 'throughput', patients who improve moving to settings of lower dependency. 


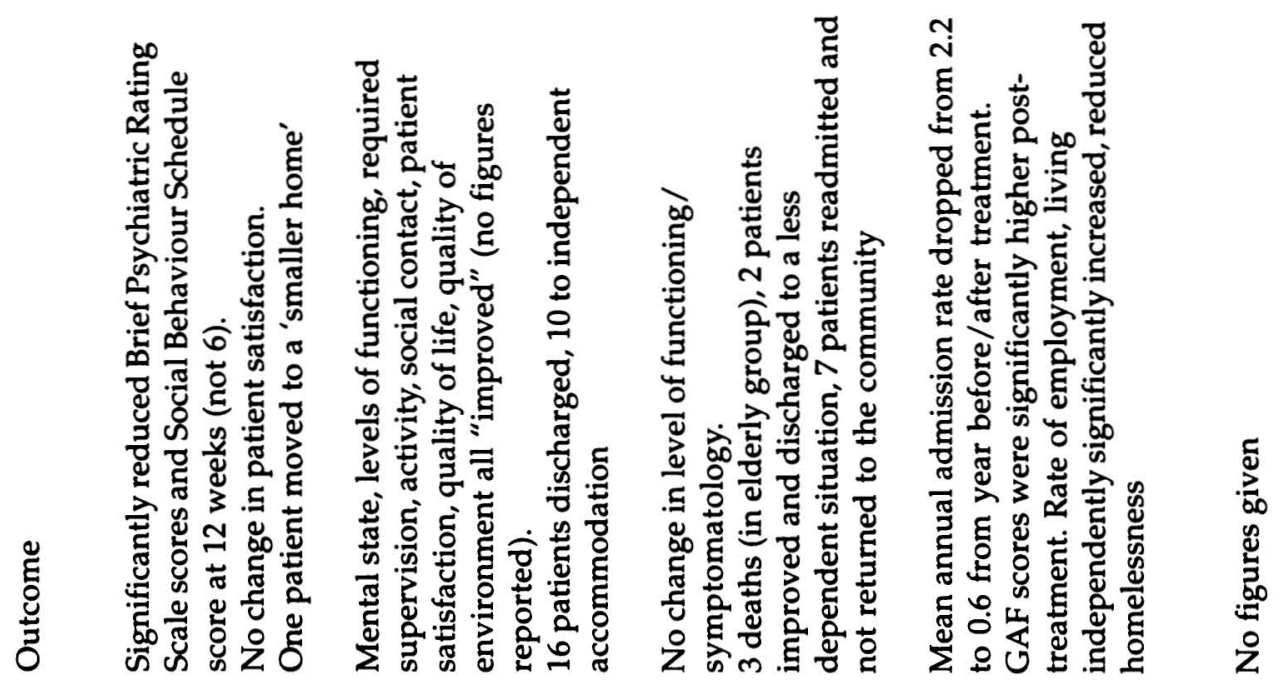

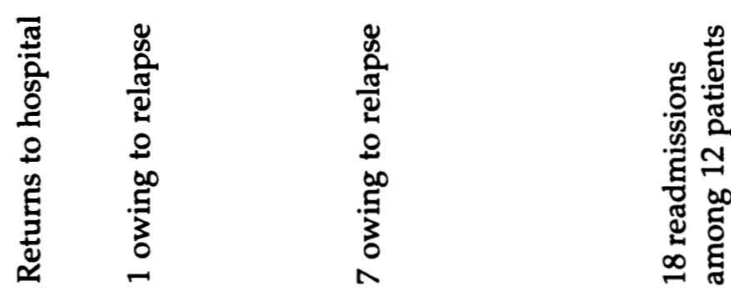

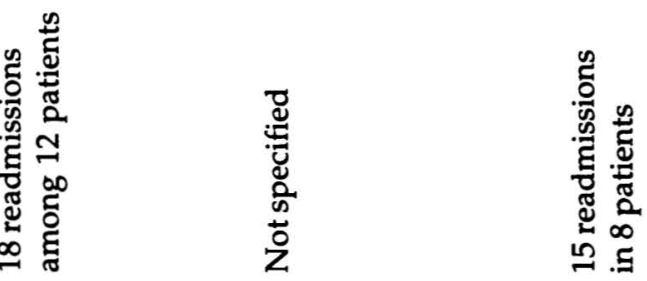

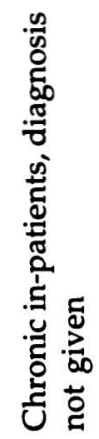
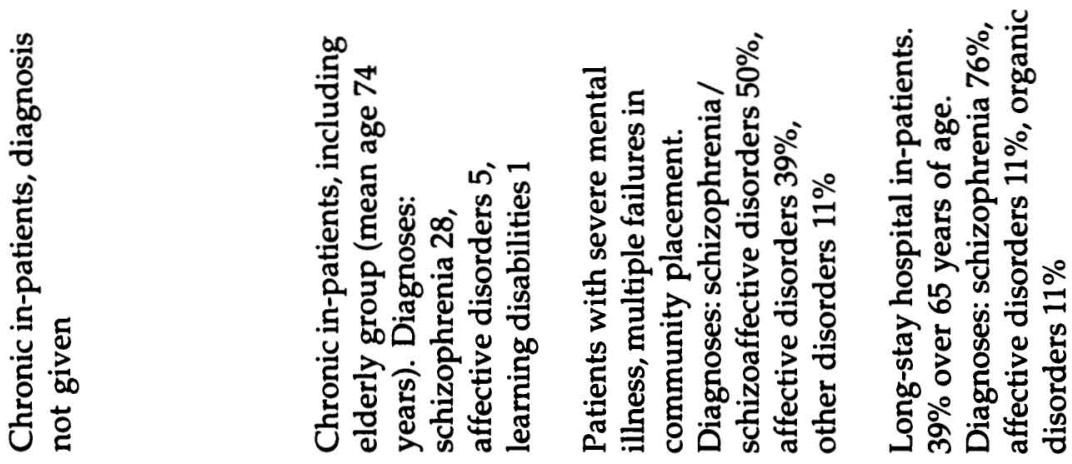

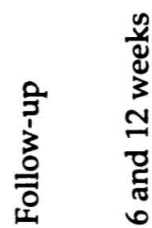

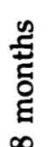

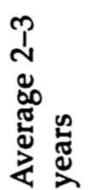

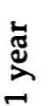

宽 岂

官

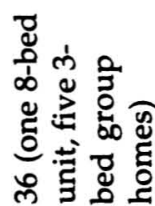

焉

章

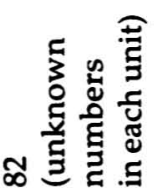

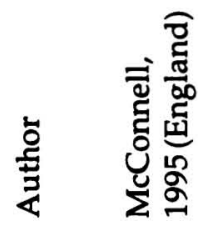

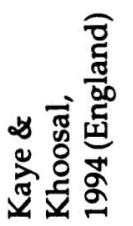

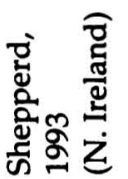

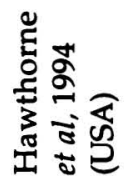

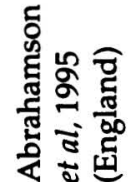



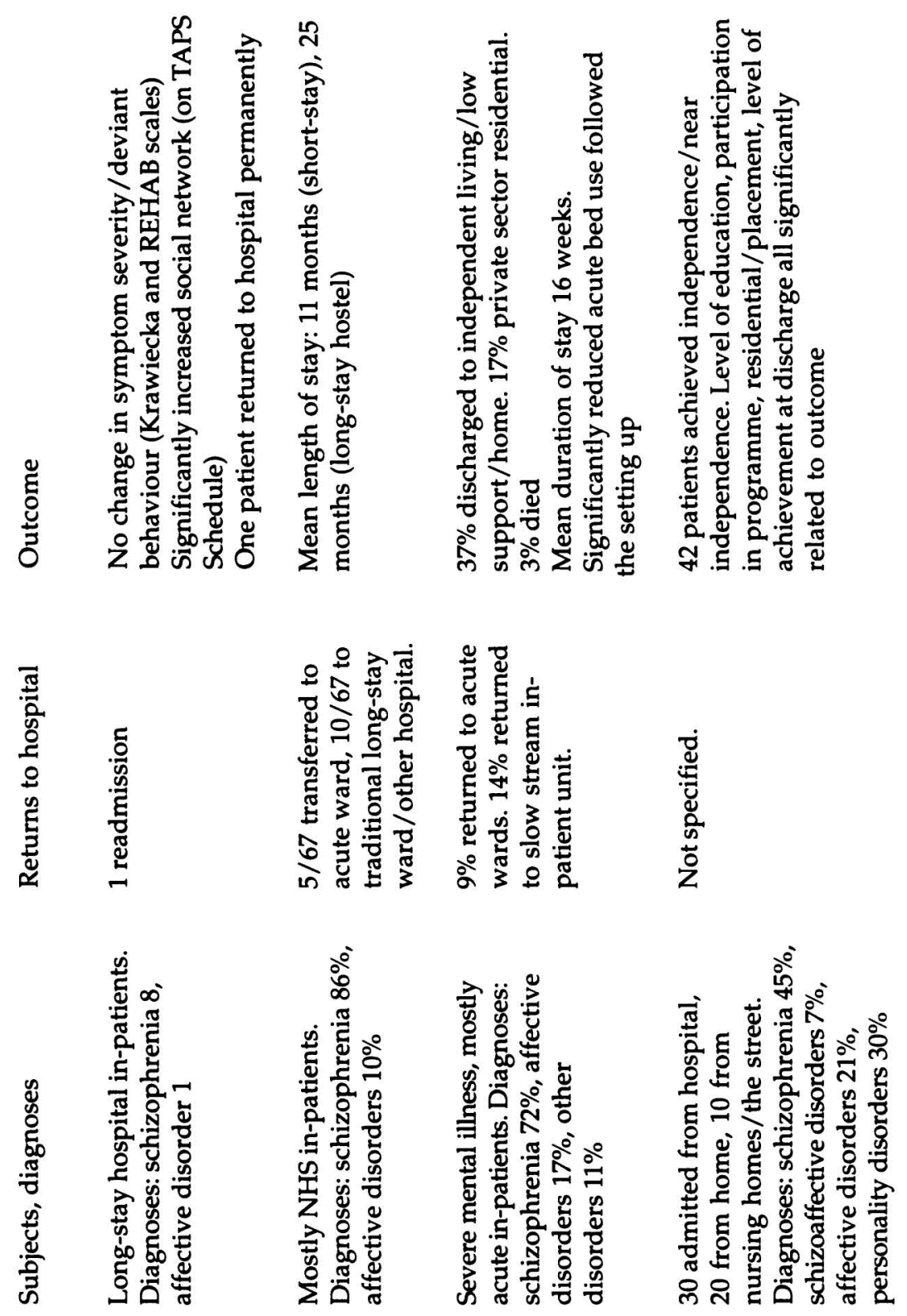

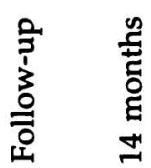

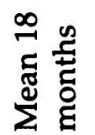
z

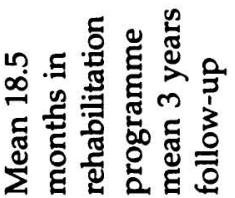

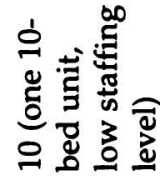

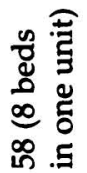

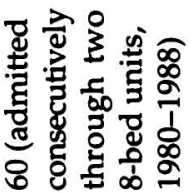

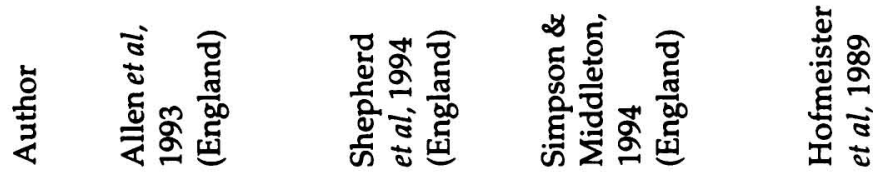


It can be seen that in many different settings, hostels have proved to be an effective environment for new long-stay patients. There is no convincing evidence of improved psychopathology in populations moved from hospital care, but there is stronger evidence regarding the positive effect on structured activity, social networks and patient/family satisfaction, improvement mostly occurring in the first three years following the placement. In most studies the majority of patients were successfully managed in hostels, although a sizeable proportion required acute readmission owing to the breakdown of care in such settings, and a somewhat smaller proportion (range $0-32 \%$, on average probably around one in ten hostel admissions) were returned to long-stay hospital care.

\section{Limitations of the research}

Most studies have described the effects of moving from long-stay or acute hospital wards to hostels, and a common flaw (from the point of view of research evidence) has been the tendency to select the most able patients, who were considered most likely to resettle successfully in hostels. Never having tested this judgement, we are unable to predict on the basis of research which patients are more likely to succeed. Furthermore, there is a tendency for those patients with the greatest psychiatric disabilities, such as antisocial and challenging behaviour, to be concentrated increasingly in the few remaining long-stay hospital wards.

In a previous review, Cournos (1987) pointed out that although community residential placements have been a leading area of expansion, there has been little scientific validation of the models. She found that environmental variables were stronger predictors of outcome than patient variables, and argued for further research to help in the development of appropriate service models. Such models should aim for optimal stimulation/stress levels, appropriate levels of structure and possibly use research concepts such as expressed emotion to assess the quality of the environment. To date, little work in this area is apparent in the literature, and this field remains an area of unmet research need.

\section{Hostels as active treatment settings for chronic illnesses}

The research to date shows that in every unit there was an active 'throughput' of patients moving to less dependent settings in the community, following placement in a hostel. The rate of throughput varied greatly, some hostels operating a 'fast-track' rehabilitation programme and averaging as little as 16 weeks' residential stay (Simpson \& Middleton, 1994), while in the majority there was a much slower turnover, averaging probably one patient per eightbed unit per year. The function of hostels evidently varies greatly, some being designed to have a more proactive rehabilitative function by taking patients primarily from acute wards early in their illness histories, whereas others are apparently set up with the aims of providing longer-term care, generally taking patients from long-stay/rehabilitation in-patient facilities. There are examples (Robson, 1993; Shepherd et al, 1994) of districts in which different hostels have been set up with explicit aims to provide active rehabilitation and continuing care.

\section{Placement following treatment in hostels}

The wide variety of final community placements (after rehabilitation through the hostel setting) described in the various studies appears to reflect the varying levels of disability on admission to hostels, and the types of special needs accommodation available in each district. Many patients actually moved to other hostels, presumably run by agencies outside the NHS, often specifically described as providing 'lower dependency care', for example having 'sleeping as opposed to waking' night cover (Shepherd et al, 1994). It is evident that the success of rehabilitation through units of this type is highly dependent on the good provision of a range of supported accommodation that meets the varying needs of patients after the period of residential rehabilitation. Strong links between the clinical team responsible for the hostel and a community rehabilitation team caring for patients in a range of other settings are important to allow an integrated and effective interplay between different parts of the psychiatric service and residential facilities.

\section{Failure of placement in hostels}

Hostels are not the answer for all patients who are difficult to place. Up to a third of placements in various studies failed and the patients generally returned to long-term hospital care. Patients with frequent or unpredictable antisocial or aggressive behaviour, or with disturbed behaviour due to combined severe mental illness and substance misuse, pose particular problems in such a 
community setting. A further group appears unable to cope with the stress of the hostel, and functions better with the "space, lower staffing levels and loose informal programmes of the traditional ward" (Shepherd, 1995). There is no clear evidence which allows us to predict which patients are more likely to succeed in a hostel.

\section{Health policy}

The value of 24-hour nursed care was recently acknowledged by the Department of Health (1996). It was described as a cost-effective alternative for patients previously accommodated on long-stay wards; such units should be "an essential component of a comprehensive psychiatric service". The NHS Executive (1996a) report on which this plan was based was broadly welcomed in a review by Holloway (1997), although he was sanguine about the view of the audit commission (NHS Executive, 1996b) that such developments would lead to a reduction in the need for acute beds, or indeed could be developed without additional resources for mental health services: while such developments may be financially viable when attached to a hospital closure programme, these relatively expensive units would require considerable additional funding in a district which had already significantly reduced bed numbers. The Department of Health (1996) has suggested a figure of $£ 25000-50000$ per bed, and recommended the provision of a minimum of 10 beds per 100000 total population.

\section{Cost effectiveness}

The question of cost effectiveness of 24-hour nursed care has been addressed in few studies, and it is difficult to consider in isolation from a total service, including total health and social care costs. The TAPS (Team for Assessment of Psychiatric Services) project (Knapp et al, 1990) found that 161 patients resettled from Friern and Claybury Hospital, largely in small staffed hostels and group homes, suffered no overall adverse clinical effects from the move, but had improved attitudes to their accommodation and reduced 'restrictiveness' in their environment. The total cost of the new model of community care was found to be lower than the previous costs of care in hospital. Individual care costs are cheaper than continuous acute in-patient treatment, but more than treatment in long-stay wards, with greater expenditure in hostels on "therapeutic" as opposed to "hotel" costs (Hyde et al, 1987). However, such direct comparisons to cost cannot account for the throughput of patients moving from hostels (and of course from hospitals) to cheaper community facilities. In real care systems, the dynamic nature of possible moves between different care settings makes costing each type of service a very difficult task.

\section{Forensic work/risk management}

At a time of increasing scrutiny of psychiatric services, the rate of inquiries into untoward incidents involving psychiatric patients is burgeoning. The practice of clinical risk assessment and management is increasingly important (with or without rationale or justification). In this context, 24-hour nursed care may have specific benefits: it allows for the community placement of patients posing a high risk of self-harm or harm to others, which could be considered safe, both in the sense of being managed and supervised, but also being contained within a process of multi-disciplinary care planning, which would be easily communicable to outside scrutiny. The slower, more carefully managed rate of transfer and rehabilitation into an open community setting may be particularly appropriate for forensic patients, for whom more sudden and radical change may be excessively stressful. While risks must be taken to help patients towards a less restrictive, more satisfying life, the hospital hostel can act as a 'half-way house' in which community placement can be partially 'tested' in a supervised environment.

\section{Relationship between hostels and general psychiatry/tertiary rehabilitation services}

In order to function well, units providing 24hour nursed care require considerable external support and monitoring by a multi-disciplinary team involving psychiatrists and psychologists, and also good access to day care facilities. They appear to be a natural focus for tertiary rehabilitation services, and in many cases appear to have worked most effectively within the structure of a strong rehabilitation service. In an interesting paper, Shepherd et al (1996) found 
that the quality of care in community homes was more determined by the personality and orientation of project leaders, and stressed the importance of ongoing review of internal management practices, staff training and the opportunity for residents to have basic choice about how and where to live. Allen et al (1989) have pointed out that care can be highly institutional in apparently forward-looking non-hospital-based settings. Staff support and training is clearly crucial.

\section{Conclusions}

Twenty-four-hour nursed care can provide community care for highly disabled, disturbed patients who would otherwise require long-term hospital provision. Its success depends on a number of factors, but there is now sufficient understanding and knowledge for such units to be integrated into standard mental health services. They should be viewed as an essential part of a range of special residential facilities to meet the varying needs of patients suffering from severe mental illness.

\section{References}

Abrahamson, D., Leitner, N. \& Sasan, S. (1995) Readmissions from registered care homes. Psychiatric Bulletin, 19, 734 736.

Allen, C. I., Gillespie, C. R. \& Hall, J. N. (1989) A comparison of practices, attitudes and interactions in two established units for people with a psychiatric disability. Psychological Medicine, 19, 459-467.

Allen, H., Baigent, B., Kent, A., et al (1993) Rehabilitation and staffing levels in a "new look" hospital-hostel. Psychological Medicine, 23, 203-211.

Anstee, B. H. (1985) An alternative form of community care for the mentally ill: Supported lodgings scheme...a personal view. Health Trends, 17, 39-40.

Cournos, F. (1987) The impact of environmental factor on outcome in residential programmes. Hospital and Community Psychiatry, 38, 848-852.

Creighton, F. J., Hyde, C. E. \& Farragher, B. (1991) Douglas House. Seven years' experience of a community hostel ward. British Journal of Psychiatry, 159, 500-504.

Department of Health (1991) Residential Needs for Severely Disabled Psychiatric Patients: The Case for Hospital Hostels. London: Department of Health.

- (1996) The Spectrum of Local Health Services for People with Mental Health Problems. London: Department of Health.

Garety, P. A., Afele, H. K. \& Issacs, D. A. (1988) A hostel ward for new long stay psychiatric patients; the career of the first 10 years - residents. Psychiatric Bulletin, 12, 183186.

Gibbons, J. S. \& Butler, J. P. (1987) Quality of life for "new" long-stay psychiatric in-patients. The effect of moving to a hostel. British Journal of Psychiatry, 151, 347-354.

Gibbons, J. S. (1986) Care of "new" long stay patients in a District General Psychiatric Unit. Acta Psychiatrica Scandinavica, 73, 582-588.
Goldberg, D. P., Bridges, K., Cooper, W., et al (1985) Douglas House: A new type of hostel ward for chronic psychotic patients. British Journal of Psychiatry, 147, 383388.

Hawthorne, W. B., Fals-Stewart, W. \& Cohr, J. B. (1994) A treatment outcome study of community-based residential care. Hospital and Community Psychiatry, 45, 152-155.

Hofmeister, J. F., Weiler, V. E. \& Ackerson, L. M. (1989) Treatment outcome in a private sector residential care programme. Hospital and Community Psychiatry, 40, 927932.

Holloway, F. (1997) 24-hour nursed care for people with severe and enduring mental illness. Psychiatric Bulletin, 21, 195-196.

Hyde, C., Bridges, K., Goldberg, D., et al (1987) The evaluation of a hostel ward: A controlled study using modified cost-benefit analysis. British Journal of Psychiatry, 151, 805-812.

Kaye, N. \& Khoosal, D. I. (1994) A ward in a street. Psychiatric Bulletin, 18, 271-273.

Knapp, M., Chisholm, D., Astin, J., et al (1990) The cost consequences of changing the hospital-community balance: The mental health residential care study. Psychological Medicine, 27, 681-692.

Lelliott, P. (1996) Meeting the accommodation needs of the most severely mentally ill. Journal of Interprofessional Care, 10, 241-247.

Mann, S. \& Cree, W. (1976) New long stay patients: A national survey of 15 mental hospitals in England and Wales 1972/ 3. Psychological Medicine, 6, 603-616.

McConnell, P. (1995) Hospital to hostel: What benefit? Psychiatric Bulletin, 19, 148-150.

National Health Service Executive (1996a) 24-hour nursed care for people with severe and enduring mental illness. Leeds: NHS Executive.

- (1996b) Priorities and Planning Guidance for the NHS: 1997/ 8. Leeds: NHS Executive.

Reid, Y. \& Garety, P. (1996) A hostel-ward for new long-stay patients: Sixteen years' progress. Journal of Mental Health, 5, 77-89.

Robson, C. E. (1993) The Vron - a short-stay rehabilitation hostel. Psychiatric Bulletin, 17, 212-214.

- (1995) Assessment of dependency level and community placement for the long term mentally ill. Psychiatric Bulletin, 19, 467-469.

Shepherd, G. (1995) The "ward-in-a-house": Residential care for the severely disabled. Community Mental Health Journal, 31, 53-69.

- (1998) Social functioning and challenging behaviour. In Handbook of Social Functioning in Schizophrenia (eds K. T. Maeser \& N. Tarrier), pp. 407-423. Hemel Hempstead: Allyn \& Bacon.

-, King, C. \& Fowler, D. (1994) Outcome in hospital hostels. Psychiatric Bulletin, 18, 609-612.

-, Muijen, M., Dean, R., et al (1995) Inside Residential Care. The Realities of Hospital Versus Community Settings. London: Sainsbury Centre for Mental Health.

-, - - - et al (1996) Residential care in hospital and in the community - quality of care and quality of life. British Journal of Psychiatry, 168, 448-456.

Shepperd, R. (1993) Readmission of long stay psychiatric patients to the acute services from hostels in the community. Psychiatric Bulletin, 17, 524-525.

Simpson, S. \& Middleton, N. (1994) Fast stream psychiatric rehabilitation after recent hospital closure. Psychiatric Bulletin, 18, 613-614.

Wing, J. K. (1971) How many psychiatric beds? Psychological Medicine, 1, 188-190.

Wykes, T. (1982) A hostel ward for "new" long stay patients: An evaluative study of a "ward in a house". Psychological Medicine Monographs Supplement 2, Cambridge: Cambridge University Press.

- \& Wing, J. K. (1982) A ward in a house: accommodation for "new" long-stay patients. Acta Psychiatrica Scandinavica, 65, 315-330. 


\section{Multiple choice questions}

1. The term "new long-stay" population was developed in the 1970s to identify:

a patients who were still receiving long-term hospital treatment after the hospital closure programme

b patients who will never be discharged from hospital

c patients who, despite modern treatment approaches, it has not been possible to discharge in the medium term from hospital settings

d patients who have stayed in hospital more than one but less than five years.

e Newly admitted patients who are likely to remain in hospital permanently.

2. 24-hour nursed care has also been termed:
a care in a "hospital hostel"
b long-stay in-patient care
c care in a psychiatric nursing home
d a "ward in a street"
e supported lodgings.

3. 24-hour nursed care is:
a an effective alternative to long-term hospital care for almost $100 \%$ of cases placed
b only of value for patients from long-stay wards
c less likely to be viable where patients present high levels of aggressive or antisocial behaviour
$\mathrm{d}$ an alternative to acute admission
e only available within the NHS.

4. Patients with the following diagnosis may benefit from 24-hour nursed care:
a organic disorder
b schizophrenia
c chronic affective disorder.
d personality disorder.
e comorbid schizophrenia/substance misuse.

5. Regarding residential service provision for patients with severe mental illness:

$\mathrm{a}$ in the past 40 years two-thirds of hospital/ hostel residential provision for the mentally ill has been closed

$b$ it is argued that 10 nursed care beds per 100000 population should be provided.

c the Government needs to commission 50000 extra beds nationally to meet current demand

$d$ there is no evidence of its effectiveness

e 24-hour nursed care is a cost-effective alternative to long-term hospital care for many long-stay patients.

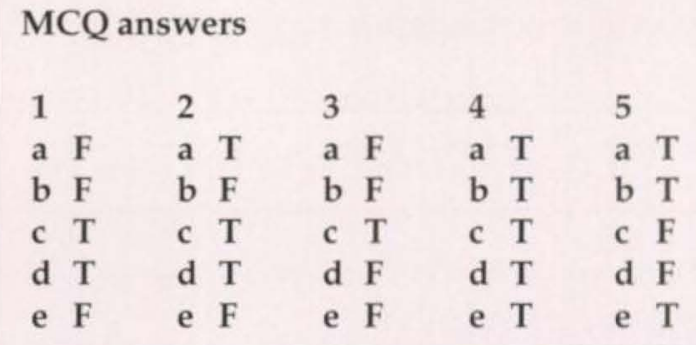

